\title{
A Novel Approach for Circular Trade Detection in Mercantile Exchange
}

\author{
Ramin Salahshoor *
}

\begin{abstract}
The derivatives market having a significant number of investors trading in futures contracts, is vulnerable to manipulation by some perpetrators. Protecting market participants from a prevalent manipulation called circular trading and providing a fair market has always been a challenging task for regulators. This kind of malpractice is represented by the trading behaviors of a group of investors who trade among themselves frequently to increase the price of the commodity and consequently make forged prosperity. This paper presents a network-based approach for detecting investors involved in circular trading in the futures market. This is done initially by constructing the daily networks of investors' trades, then, extracting all trade cycles of various lengths from these daily networks to arrive at the group of initial suspicious cycle traders. Finally, in order to exclude investors who are randomly involved in suspicious cycles, price fluctuations over time were analyzed. The proposed approach has been conducted on real data from Iran Mercantile Exchange (IME) and as a warning system, has succeeded in detecting anomalous traders effectively.
\end{abstract}

Keywords: Circular trading; anomaly detection; trade networks

\section{Introduction}

Illegal or misconduct that appears in the financial market includes behaviors such as financial fraud, insider trading, bribery, self-dealing, money laundering, tax evasion, corruption and a specific category of mal-practices like broker embezzlement, Pyramid scheme, Ponzi scheme, high yield investment and circular trading known as market manipulation. Market manipulation which is the target field in this study involves individuals or a group of people attempting to interfere with a fair and orderly market to gain profit (Reurink, 2016).

Circular trading is still one of the biggest concerns of investors and regulators in the capital markets, although many efforts have been made to reduce the effects of such malpractice on the markets reliability. Circular trading is described as the trading behavior of a group of accomplice investors who trade among themselves frequently to force an increasing trend in the price of the commodity, as well as incentivizing other traders to follow them and consequently create a false market. These accomplice investors are in fact herding the other investors into trading based on a new inflated price (Choi \& Skiba, 2015). Some of the researchers like Vo and Phan (2017) has also attempted to survey the presence of herding behavior and its underlying reasons. Their findings indicate that herd behavior is evident during high and low trading volume days and does not exhibit during public meltdown. They also observed that it is stronger in post-crisis than pre-crisis. But, according to investigations, it is not complicated for a group of influential investors

*MSc in Information Technology from University of Tehran. E-mail: raminsalahshoor@gmail.com 
to manipulate the market and herd the other investors, but the real challenge for them is to make the manipulations protable.

To facilitate fair trade, the competent authorities keep applying various guidelines to be followed by all participants in market activities, as well as developing smart systems to monitor their actions. These prevalent surveillance systems which usually use machine learning techniques are generally unable to detect minute by minute occurrences of complex types of mal-practices like circular trading because they naturally need access to the history of each investor's transactions which are mostly classified and inaccessible at the moment. In fact, they cannot analyze short-term trading data in the available limited time. Such systems could be preventive provided that they detect circular trading premature.

In this paper we present a novel network-based approach that is able to discover anomalous traders. This approach is applied to data from Iran Mercantile Exchange (IME) market. IME is a commodities exchange located in Tehran, Iran. Various products and commodities are listed and traded in IME including industrial products and commodities, oil products and petrochemicals, agricultural, and futures contracts of gold coin. For the purpose of this research, data from the gold coin futures contracts have been used.

The remainder of this paper is organized as follows: In Section 2, a survey of some related works is provided. In Section 3, the basic approach for detection of anomalous traders, including data preparation, extraction of all trade cycles, investigation of transactional behaviors of traders involved in such cycles has been described. Section 4 provides experimental analysis and elaborates on the results, as well as suggestions for future research directions. Finally, section 5 concludes the paper.

\section{Related Work}

Graph structure is an expressive data structure model which is able to examine relationships among objects in applications such as transport networks, biological networks, computer and telecommunication networks and social networks. Recently, graph based approaches and algorithms have been considered as a powerful tool to analyze financial networks like the network formed by investors' transactions (Lillo \& Valdés, 2016).

There are many different models of graph-based methods being used in nancial networks, each one assessing a dierent aspect of connectivity and dynamism. In some approaches, the network is defined such that there is no links or communication between nodes of the same kind such as sellers with other sellers, or buyers with other buyers. Hence this leads to creation of bipartite graphs where sellers or assets are placed in one part and buyers or investors, in the other part (Dehmamy, Buldyrev, Havlin, Stanley, \& Vodenska, 2016; Hemenway \& Khanna, 2017; Sun, Qu, Chakrabarti, \& Faloutsos, 2005).

Moreover, it is possible to categorize all nodes to three groups such as fraudster, accomplice and normal (Chau, Pandit, \& Faloutsos, 2006) and as fraudsters and their accomplices probably form a bipartite graph, a Markov Random Field (MRF) model could be built from the transaction history amongst all traders, then the belief propagation algorithm is applied to calculate the probabilities of fraudster, accomplice and normal user for each node in the resulted graphs. 
Another set of methods (Jallo, Budai, Boginski, Goldengorin, \& Pardalos, 2013; Liu \& Chi, 2012; Li et al., 2015) is using other coefficients and indices like correlation coefficient and assortative index to construct the network's graph by measuring the similarity between stocks; clustering dependent ones that tend to trade only with one another. These approaches also investigate the network's components and their impacts on market dynamics which are mostly due to fluctuations in financial variables like turnover and volatility.

In recent years, a new model of graph-based methods has been used in a few studies. This category of approaches is based on extracting building blocks of graphs like cliques, motifs or other topological structures and scrutinizing them to find anomalous groups or behaviors. Wang, Zhou, and Guan (2012) proposed a method to detect hidden collusive cliques of traders collaborating to manipulate the market and mislead other investors to maximize their personal benefits. They calculated correlation between any pairs of time series of orders in the futures market and then combined the daily weighted graphs into an integrated graph in which the fully connected subgraphs (or cliques) are extracted as the suspect collusive cliques. Jiang et al. (2013) investigated three specific abnormal motifs comprised of self-loop, two-node loop and two-node multiple arcs and their evolution in a time-varying network of traders' transactions. They further studied the dynamics of financial variables around the transactions associated with the mentioned motifs. Xie, Li, Jiang, and Zhou (2014) constructed dependence networks from weighted friendship networks of avatars in virtual societies of a massively multiplayer online role-playing game. They examined the evolution of thirteen kinds of triadic motifs in dependence networks and distribution of their occurrence frequency and relations between their counts. Bender, Fineman, Gilbert, and Tarjan (2016) propose two algorithms, one suited for sparse graphs and the other to dense graphs to address the problem of detecting a cycle in a directed graph that grows by arc insertions, and the related problems of maintaining a topological order and the strongly connected components in such graphs.

To the best of our knowledge, there is no work that develops an approach to extract trade cycles of various lengths as topological structures from traders' network of future contracts as proposed in this paper. The novelty of this approach is that after extracting the frequency of each daily trade cycle over a period of time, it will then compare and trace their behavior over that time period to ensure market manipulation. Results are later verified by the experts.

\section{Data Set}

In the futures market, there are several types of data, including order records, positions and match trades. Order records consist of information about investors such as their decisions to buy or sell, the volume and price of their requested commodity as well as the corresponding times. The exchange market's internal computer system then compares all the order records to find pairs with the same requested value of commodity, while one is for a seller and the other, a request to buy. The system will then change the investors' positions and report their orders as an integrated trade record in the match trades data. 
The dataset under investigation is the match trades data related to four future contracts of gold coin traded in Iran Mercantile Exchange (IME) during 618 trading days from April 2013 to January 2016. The data set contains 1,600,000 match trades records and 2095 market participants.

Each match trades record contains the following information: contract id, that could be used as a unique key in the database, number of gold coin packs, as the contract size (each pack contains 10 gold coins), price of each gold coin in that specific contract, encrypted identity of the brokers and investors and the last column is also time stamp of the contract.

As to brokers and investors, commodity broker is a firm or individual who executes orders to buy or sell commodity contracts on behalf of the clients and charges them a commission. An individual who trades for his own account is called a trader or investor. A sample view of this dataset is illustrated in Table 1 below.

Table 1

Descriptive statistics of stock returns

\begin{tabular}{|c|c|c|c|c|c|c|c|}
\hline \multirow[t]{2}{*}{ Contract-Id } & \multirow[t]{2}{*}{ No. of Gold packs } & \multirow[t]{2}{*}{ Price } & \multicolumn{2}{|c|}{ Seller } & \multicolumn{2}{|c|}{ Buyer } & \multirow[t]{2}{*}{ Time } \\
\hline & & & Investor & Broker & Investor & Broker & \\
\hline 1354872 & 2 & $1,078,540$ & 0x11FB3AE & 0x5G4E27D & 0x9F8EAB6 & 0x8A6B18F & $\begin{array}{c}2015-01-13 \\
11: 00: 27: 387\end{array}$ \\
\hline 1367529 & 3 & $1,046,890$ & 0x1E345DB & 0x723F4E6N & 0x87C43B2 & 0x243D5A7 & $\begin{array}{c}2 / 17 / 2015 \\
14: 23: 37: 522\end{array}$ \\
\hline
\end{tabular}

\section{Methodology}

In this section, the details of the various steps taken to detect anomalous traders, is described. First of all, the daily graphs of traders need to be constructed in order to be examined by three main modules of the proposed approach. We have called these modules Trade Cycle Extraction (TCE), Suspicious Cycles Selection (SCS) and Transactional Behaviors Evaluation (TBE) which have to be executed respectively. In TCE module, all cycles of various lengths are extracted from all daily graphs. SCS module is in charge of selection of initial cycles that are suspicious to be anomalous regarding the defined conditions of being anomalous. In TBE module as the final step, transactional behaviors of the traders involved in the suspicious cycles selected by SCS module are investigated to verify their suspicious activities.

\section{Graph Construction}

To construct the trading graph, it is essential to determine the types of nodes and edges. In this study, we consider each investor as a node and each transaction as a directed and weighted edge from seller to buyer. The weight of the edge is shown by an ordered pair in which the first element contains the price of the gold coin in that specific transaction and the second element holds the time of the transaction.

In the future contracts of gold coin (or any other commodity), investors are allowed to trade with one another without any limits, therefore we will have a multi-edged graph. On the other hand, investors cannot trade with themselves, hence there are no self-loops 
in the graph. On the whole, the resulted graph is a multi-edged directed and weighted graph without self-loop, similar to the sample graph depicted in figure 1. To clarify, trader(node) number 10 sells a gold coin to trader(node) number 14 for the price of 1.113 .400 at 11:03:27 AM.

As it is likely for an investor to trade in one day and then not participate in the market for several days, therefore, each trading day is investigated independently and daily trade graphs are constructed and analyzed. Hence, we will have 618 graphs, each one being created based on a specific day's transactions.

\section{Figure 1}

A sample of daily trade graphs from the IME - a multi-edged directed and weighted graph without self-loop

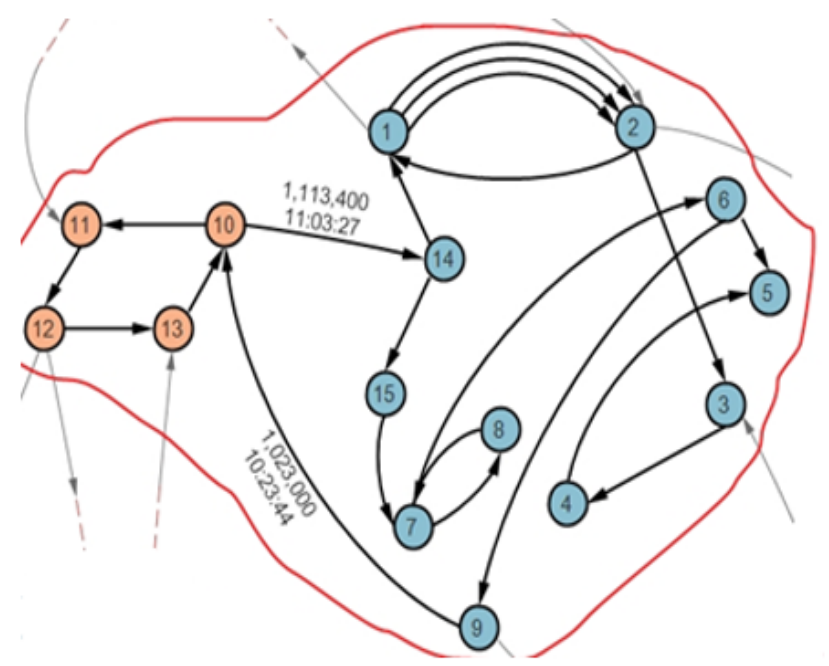

\section{Trade Cycle Extraction (TCE)}

A circular trade as referred to here is the same as a cycle in the corresponding directed trade graph. To detect anomalous traders who are involved in circular trades, we need to extract all cycles of various lengths from all 618 constructed graphs of daily trading networks.

Figure 2 shows a real circular trade detected in one of the daily graphs of the dataset. As it is clear, investor A buys 10 packs of gold coin for the base price of one million Toman (Iranian currency) and then sells two of these packs to one of his accomplices, investor B, with a $4 \%$ increase in price. Then, investors B, C and D follow the same procedure and sell those two packs among themselves, increasing the price by approximately $5 \%$ (as the maximum limit that each investor is allowed to register a new order by) each time until it reaches back to investor A. So, investor A, who sold those two packs to investor B in the first place, now becomes the buyer after a cycle of trades, and buys them again from 
investor D. He will then sell them, with about $25 \%$ increase in price, to an investor who has been impressed by the market's fake prosperity. Finally, investor A participates in the market affected by a fake prosperity and sells the remaining of those 10 packs of gold coin which he has bought initially and not traded yet.

Consequently, investors A, B, C and D are likely to be anomalous traders who manage to benefit from the false increase of commodity price in the market. But according to experts' opinion, due to instinct of future market that is not enough to label them as certain anomalous traders because this kind of trades could happen occasionally and randomly. Therefore, we introduced the following step, Suspicious Cycles Selection, to analyze them deeply.

Figure 2

A real circular trade detected in one of the daily trade graphs

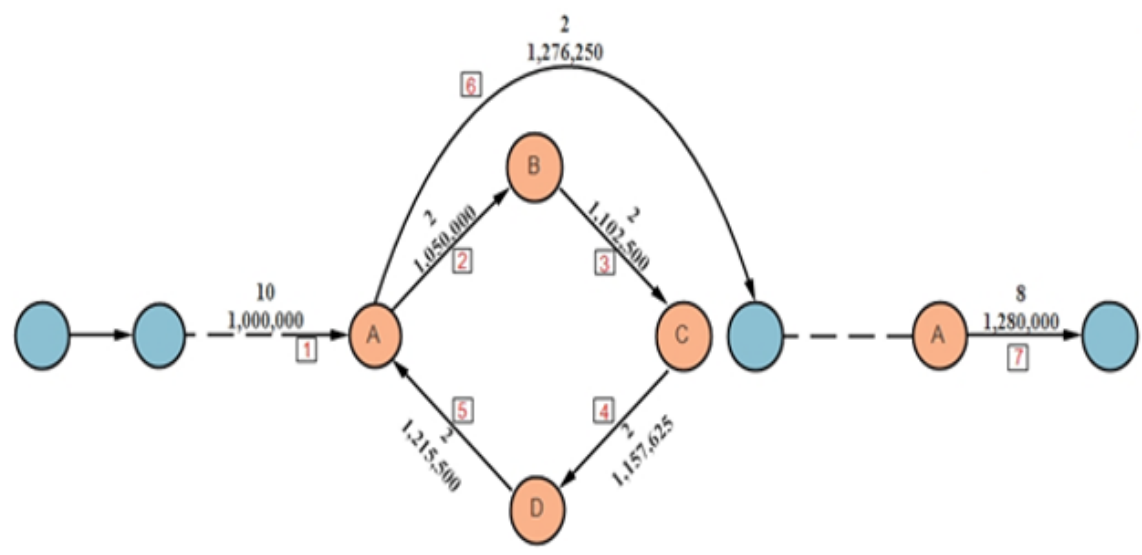

After extraction of all cycles from the given daily trade graphs, it was noticed that there were no cycles with more than 5 nodes involved. Hence, all retrieved cycles were cycles with 3, 4 or 5 nodes like those shown in figure 3.

\section{Suspicious Cycles Selection (SCS)}

Needless to say, when we are studying a market with more than 1,600,000 trades and then we construct this market's graph, we should expect to encounter too many cycles as one of the most common topological structures of its graph. So we have to sift these cycles to exclude normal ones that have been shaped randomly and on the other hand to select suspicious cycles for further investigation.

In Our study ninety-nine cycles became discovered that among them there were 65 cycles without any abnormal behavior and appearing just once so that we put them aside. But also there were 34 cycles with two abnormal properties that made us label them as initial suspicious cycles for extra examination. The first abnormal behavior was observing 25 cycles being repeated in several different trading days. For example, we found two fivenode cycles with four occurrences during one week. At first glance, that seemed logical 
to consider this behavior abnormal because a group of anomalous traders undoubtedly would not abandon the market after doing circular trade just for once, and tend to appear again and gain more profit from consequent circular trades.

And the second abnormal behavior was finding nine cycles that had identical subsequences inside one another, i.e. same order of nodes in the cycle. As it is indicated in figure 3, the subsequence $X-Y$ has appeared in three different cycles by different lengths.

Figure 3

Same subsequence of traders $(X-Y)$ in three different cycles of different lengths
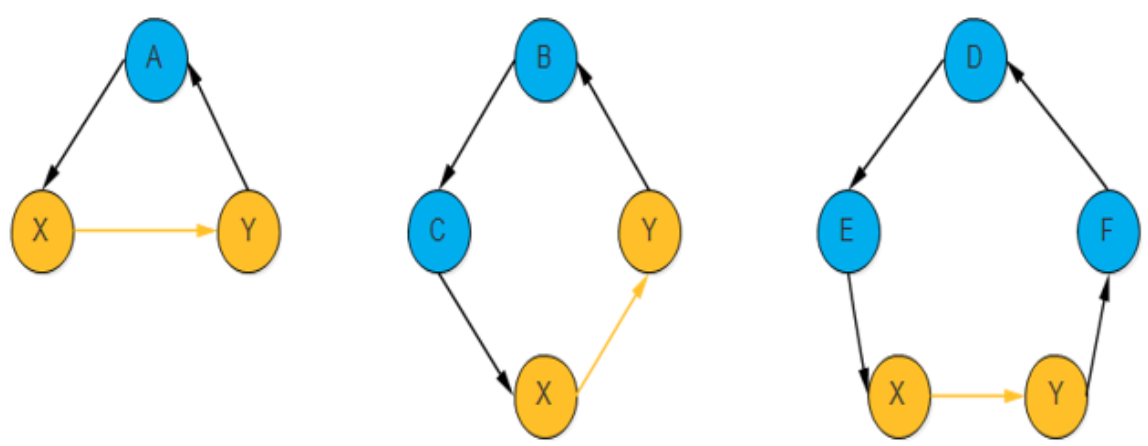

Eventually, these 34 initial suspicious cycles and traders creating them are delivered to next module, Transactional Behaviors Evaluation, as its input. Detailed information of these cycles are listed in the fourth column of table 3 at the result section.

\section{Transactional Behaviors Evaluation (TBE)}

After separating those 34 initial suspicious cycles, based on their repetition and similar subsequences, from the rest of cycles by the SCS module, we decided to examine individually the transactional behaviors of the traders involved in them. The purpose of this step is stemmed from two probabilities. First, we could not forget that in a dynamic market like future market it is highly likely to have traders taking part randomly in some malicious circular trades even when we find the most suspicious cycles. And the second purpose is calculation of these traders' profit or loss.

Given market regulators' opinion, although contributing to a circular trade and earning nothing or even making loss in return could mean colluding with other traders but does not justify these traders' malicious behavior and they would better to be exempted from later punishment defined in market's bylaw. Therefore, we employed this step to know more about these traders' intention of playing a part in cycles and to discriminate between those who are making profit and those who earn nothing at the end of the cycle.

In order to examine independently the transactional behaviors of such suspicious traders, we need to plot the time series of the price fluctuations of the commodity (Gold Coin) relating to the corresponding trading days that target investor was trading in. Each 
time series is then divided into three time windows and each one is analyzed separately that we elaborate on this procedure in the following.

According to experts' knowledge, there is a condition to discern a real malicious circular trade from a simple cycle which has been made randomly and unintentionally. As was explained in the trade cycle extraction(TCE) step, traders who commenced a suspicious circular trade, in fact the first nodes of cycle, must have participated in the market during the first time window (The green part in Figure 4) and bought some commodity (packs of gold coin) with a price cheaper than the average price of the day that this average price needs to be calculated for each trading day. Also within this time window market witnesses just some slight fluctuations in price and there is not any extraordinary rise or fall.

Then, during the second time window (The orange part in Figure 4) it is expected that the price rises significantly (above the average fluctuations) due to the frequent trades among teamed-up investors. These investors are trading a small amount of commodity (gold coin packs), bought within the first time window, as a leverage to create a false market.

\section{Figure 4}

A sample of two daily time series of price fluctuations in the market corresponding to an anomalous (23rd May 2015) and a normal (14th Feb 2015) trading day

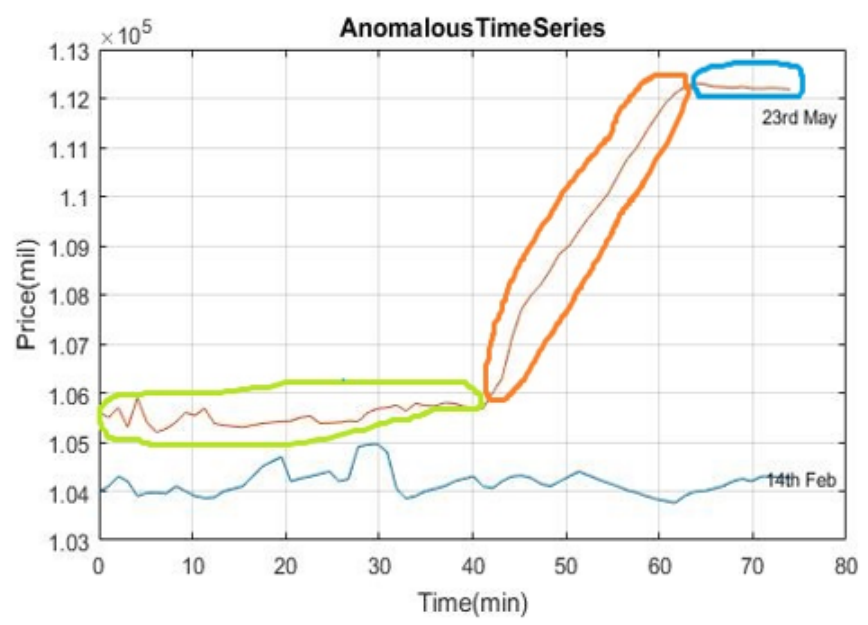

Lastly, through the third time window (The blue part in Figure 4), we are now faced with a market following a trend of price increase which is fake. It is in this time window that the initial member of the suspicious trade cycle, attempts to sell the rest of the batch commodity, which had not been traded during the second time window, based on the new soared price. This is something that must be verified by analyzing each of the consecutive time windows per trading day. Hence after this step, those trade cycles that fit such activities are verified and their members are introduced as potential cycle traders. 
Figure 4, shows a real instance of time series for the day when a circular trade occurred, plus a sample of a normal day without any circular trade to compare. It is worth noting that the time series plot contains all the (matched) price fluctuations of the commodity (in this case gold coin future contracts), from the moment the market opens until the end of the day.

As an example, the time series for 23rd of May 2015 is highlighted with the three time windows clearly shown in Figure 4. The first time window (The green part) covers the time from market opening to just before a circular trade occurs (around 40 minutes after market opening). The second time window (The orange part) covers the span of time while circular trade is occurring. As was predicted and can be seen from the results, it shows an increasing trend in the price. The third time window (The blue part), then shows the interval between the moment that circular trade terminates until the end of that trading day. For comparison, the time series for 14th of Feb 2015 is also shown on the same figure, as an example of a normal trading day along with the usual price fluctuations.

Finally, after time series evaluation of the 34 suspicious cycles found in the SCS step of the approach that 12 samples of them is presented in Figure 5, we observed that some of these cycles (four 3-node cycles, four 4-node cycles and two 5-node cycles, 10 cycles in total) did not manage to create a sharp rise in the commodity price, and hence create a false market.

\section{Figure 5}

12 samples of anomalous and normal time series as our initial extracted suspicious cycles

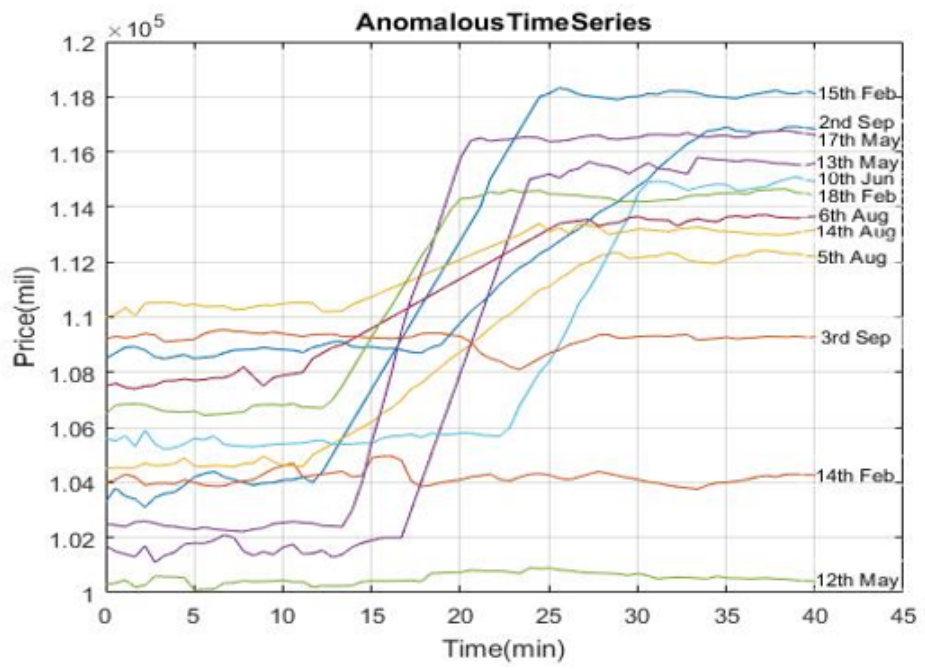

Therefore, seven 3-nodes cycles, nine 4-nodes cycles and eight 5-nodes cycles, 24 cycles in total, succeeded in faking a prosperity in the market and shaping a new trend of price based on circular trading. In other words, 97 traders who were involved in the mentioned 24 trade cycles, met the defined conditions of being anomalous. Hence, they are introduced as potential anomalous traders to market regulators for further probe. 


\section{Time Complexity}

Computational complexity of the proposed approach is linear with respect to the complexity of three main modules of the approach namely Trade Cycle Extraction (TCE), Suspicious Cycles Selection (SCS) and Transactional Behavior Evaluation (TBE) which are represented by the pseudocode given below in table 2 .

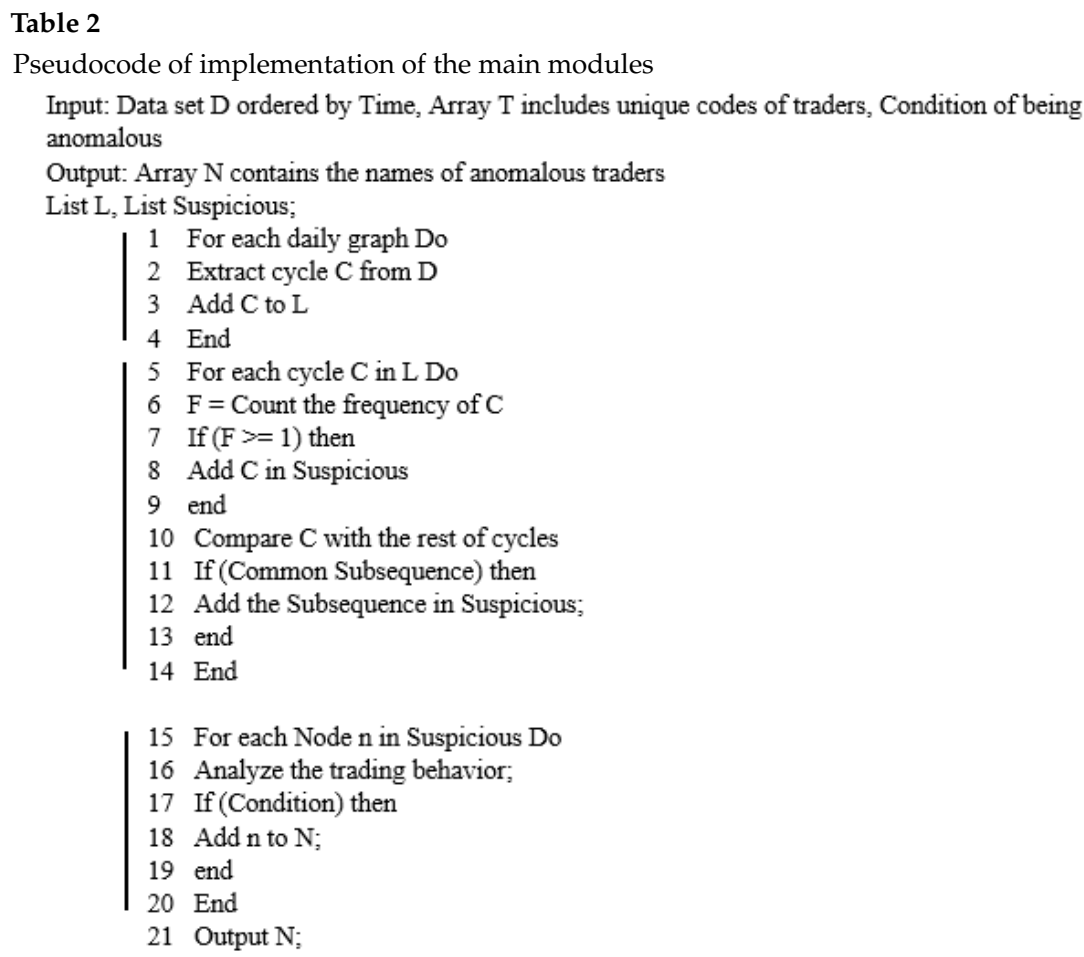

According to the above algorithm, the CE module can be calculated in $O(n)$. Besides, SCS module and TBE module add a similar $\mathrm{O}(\mathrm{n})$ to the complexity of the proposed approach, leading to an overall linear complexity. Moreover, there are some constants such as number of trading days or number of investors in a single day that will not affect the order of computational complexity.

\section{Results}

In this section we report on results obtained from applying our approach to real datasets, collected from Iran Mercantile Exchange (IME) during 618 trading days from April 2013 to January 2016. We ran the analysis on the 1,600,000 match trades records and 2095 market participants. 
In table 3, a breakdown of the all examined cycles extracted from daily graphs by the TCE module is given.

\begin{tabular}{|c|c|c|c|c|}
\hline Cycle Length & $\begin{array}{c}\text { Number of } \\
\text { Extracted Cycles }\end{array}$ & $\begin{array}{c}\text { Number of Unique Nodes } \\
\text { Involved in Cycles }\end{array}$ & $\begin{array}{l}\text { Repeated Cycles and cycles } \\
\text { with similar subsequences }\end{array}$ & Nodes Overlap \\
\hline \multirow{3}{*}{3} & \multirow{3}{*}{47} & \multirow{3}{*}{133} & $\begin{array}{l}6 \text { cycles with } \\
2 \text { occurrences during } 1 \text { month }\end{array}$ & \multirow{3}{*}{$6 \%$} \\
\hline & & & $\begin{array}{l}2 \text { cycles with } \\
3 \text { occurrences during } 2 \text { consecutive days }\end{array}$ & \\
\hline & & & $\begin{array}{l}3 \text { cycles with } \\
\text { similar subsequences }\end{array}$ & \\
\hline \multirow{3}{*}{4} & \multirow{3}{*}{35} & \multirow{3}{*}{127} & $\begin{array}{l}8 \text { cycles with } \\
2 \text { occurrences during } 1 \text { month }\end{array}$ & \multirow{3}{*}{$10 \%$} \\
\hline & & & $\begin{array}{l}3 \text { cycles with } \\
3 \text { occurrences during } 10 \text { days }\end{array}$ & \\
\hline & & & $\begin{array}{l}2 \text { cycles with } \\
\text { similar subsequences }\end{array}$ & \\
\hline \multirow{3}{*}{5} & \multirow{3}{*}{17} & \multirow{3}{*}{66} & $\begin{array}{l}4 \text { cycles with } \\
2 \text { occurrences during } 2 \text { consecutive days }\end{array}$ & \multirow{3}{*}{$28 \%$} \\
\hline & & & $\begin{array}{l}2 \text { cycles with } \\
4 \text { occurrences during } 1 \text { week }\end{array}$ & \\
\hline & & & $\begin{array}{l}4 \text { cycles with } \\
\text { similar subsequences }\end{array}$ & \\
\hline Total & 99 & 176 & 34 & \\
\hline
\end{tabular}

According to results in table 3, all the extracted cycles have less numbers of unique nodes than expected. For example, for 47 cycles of length 3 nodes, one would expect 141 unique nodes by multiplying 47 by 3 , yet there were 133 unique nodes, meaning that 8 nodes or approximately $6 \%\left(\frac{8}{133} * 100\right)$ of unique nodes have been repeated in some of the cycles. Also for 35 four-node cycles in the third row of the table we expected to have 140 unique nodes while we just saw 127 unique nodes implying that 13 nodes were appearing in several cycles. And finally for 17 five-node cycles in the fifth row we had a relatively similar situation and found 66 unique nodes which among them 19 nodes were repetitive.

Accordingly, the percentage of similar nodes which have been repeated in several cycles, shown in the last column of table 3 as Nodes Overlap, increases from 6\% in 3-node cycles to $28 \%$ in 5-node cycles. In fact, this increase is proving that larger cycles are more private than smaller ones and traders who are involved in them are familiar with one another, thereby reducing the likelihood of strangers entering their cycle and hence disturbing their malpractice. In other words, since creation of small cycles needs less organization due to fewer number of traders participating in them, they are more convenient to be shaped and more likely to have traders randomly involved in. Therefore, the larger the cycle is, the more repetitive and anomalous trader there is. Table 4 reports the final results of applying the proposed approach to the real data set of mercantile exchange which can confirm its effectiveness in detecting anomalous traders. At the first row of the table it 
is mentioned that we discovered 47 3-node cycles so that after checking on their abnormal behaviors we selected 11 cycles as initial suspicious cycles. Then, we evaluated time series of these cycles and found just 7 cycles faking a prosperity in the market and we introduced 21 traders shaping these 7 cycles as fraudsters to the market regulators. Finally, they confirmed 12 of them as real anomalous traders. Also, 21 traders from the 4-node anomalous cycles and 26 traders from 5-node anomalous cycles were confirmed as real fraudsters by the experts.

Overall, more than $60 \%\left(\frac{59}{97} * 100\right)$ of the introduced anomalous traders were real fraudsters, which with respect to the linear complexity of the proposed approach and its duty as an alarm system, is acceptable.

\begin{tabular}{lcccc}
$\begin{array}{l}\text { Table } 4 \\
\text { Analysis of the final output of the applied approach }\end{array}$ & & \\
\hline $\begin{array}{l}\text { Length of } \\
\text { Cycle }\end{array}$ & $\begin{array}{c}\text { Number of } \\
\text { Extracted Cycles }\end{array}$ & $\begin{array}{c}\text { Number of } \\
\text { Suspicious Cycles }\end{array}$ & $\begin{array}{c}\text { Number of } \\
\text { Anomalous Traders }\end{array}$ & $\begin{array}{c}\text { Number of } \\
\text { Confirmed }\end{array}$ \\
\hline 3 & 47 & 11 & 21 & 12 \\
4 & 35 & 13 & 36 & 21 \\
5 & 17 & 10 & 40 & 26 \\
Total & 99 & 34 & 97 & 59 \\
\hline
\end{tabular}

In this study, the verification process has been done by the market regulators and experts and the final word on whether introduced anomalous traders are fraudsters or not belongs to them. Because in the financial industry, it is against the rules to disclose market data or even history of investors, hence there are not enough information for researchers to label the data and verify the results. However, such results are very valuable to the market supervision division, which gives them some factual information on target or potential fraudsters for more investigation, rather than going through a huge amount of trading data.

\section{Conclusion}

In this research, a graph-based approach has been proposed to detect anomalous traders who are manipulating the price of the future contracts by circular trading in mercantile exchange. The suggested approach extracts all the cycles of various lengths from the defined multi-edged directed graph of investors. Then a group of suspicious cycles become selected based on the specific features of cycles to plot time series of price fluctuation relates to the days that they have been extracted from. Time series of some cycles showed their mal-practice meeting the rule of being anomalous and finally those traders who have been involved in these anomalous cycles are introduced to market regulators for further probes.

In addition to the capability of suggested approach to both construct graphs of investors' networks and extract suspicious cycles from them as circular trades, it is also able to evaluate investors involved in suspicious cycles by time series analysis, prior to announcing them as anomalous investors to the market regulators. 
As future work, we are considering to improve our approach to a real-time version to be able to warn market regulators as soon as a trade cycle is detected, whereas presently it is bound to have access to all records of a trading day together. Moreover, the present approach has only been applied to future contracts in mercantile exchange while other markets and financial instruments are severely suffering from circular trading and our approach with an alteration would be operative for them. 


\section{References}

Bender, M. A., Fineman, J. T., Gilbert, S., \& Tarjan, R. E. (2016). A new approach to incremental cycle detection and related problems. ACM Transactions on Algorithms (TALG), 12(2), 1-20.

Chau, D. H., Pandit, S., \& Faloutsos, C. (2006). Detecting fraudulent personalities in networks of online auctioneers. Springer.

Choi, N., \& Skiba, H. (2015). Institutional herding in international markets. Journal of Banking E Finance, 55, 246-259.

Dehmamy, N., Buldyrev, S., Havlin, S., Stanley, H. E., \& Vodenska, I. (2016). Crises and physical phases of a bipartite market model.

Hemenway, B., \& Khanna, S. (2017). Sensitivity and computational complexity in financial networks. Algorithmic Finance, 5(3-4), 95-110.

Jallo, D., Budai, D., Boginski, V., Goldengorin, B., \& Pardalos, P. M. (2013). Networkbased representation of stock market dynamics: An application to American and Swedish stock markets. In Models, algorithms, and technologies for network analysis. Springer.

Jiang, Z.-Q., Xie, W.-J., Xiong, X., Zhang, W., Zhang, Y.-J., \& Zhou, W.-X. (2013). Trading networks, abnormal motifs and stock manipulation. Quantitative Finance Letters, $1(1), 1-8$.

Li, M.-X., Jiang, Z.-Q., Xie, W.-J., Xiong, X., Zhang, W., \& Zhou, W.-X. (2015). Unveiling correlations between financial variables and topological metrics of trading networks: Evidence from a stock and its warrant. Physica A: Statistical Mechanics and its Applications, 419, 575-584.

Lillo, F., \& Valdés, R. (2016). Dynamics of financial markets and transaction costs: A graph-based study. Research in International Business and Finance, 38, 455-465.

Liu, X. F., \& Chi, K. T. (2012). Dynamics of network of global stock markets. Accounting and Finance Research, 1(2), 1-12.

Reurink, A. (2016). Financial fraud: A literature review (Tech. Rep.). MPIfG Discussion Paper.

Sun, J., Qu, H., Chakrabarti, D., \& Faloutsos, C. (2005). Relevance search and anomaly detection in bipartite graphs. ACM SIGKDD Explorations Newsletter, 7(2), 48-55.

Vo, X. V., \& Phan, D. B. A. (2017). Further evidence on the herd behavior in Vietnam stock market. Journal of Behavioral and Experimental Finance, 13, 33-41.

Wang, J., Zhou, S., \& Guan, J. (2012). Detecting potential collusive cliques in futures markets based on trading behaviors from real data. Neurocomputing, 92, 44-53.

Xie, W.-J., Li, M.-X., Jiang, Z.-Q., \& Zhou, W.-X. (2014). Triadic motifs in the dependence networks of virtual societies. Scientific Reports, 4(1), 1-8. 